\title{
A new slowly pulsating subdwarf-B star: HD 4539
}

\author{
C. Schoenaers, A.E. Lynas-Gray \\ Department of Physics, University of Oxford, Keble Road, Oxford OX13RH, United \\ Kingdom
}

\begin{abstract}
We report the spectroscopic discovery of slow pulsations in the subdwarf-B (sdB) star HD 4539. It is amongst the brightest sdB stars and, as such, has been well studied. Its temperature and gravity would place it squarely where the so-called "Betsy" stars (long-period sdB pulsators) are found, and this has been confirmed by the discovery of line-profile variations reported in this paper for the first time. As periodogram analyses of radial velocity curves obtained in 2005 and 2006 yield several significant frequencies, line-profile variations are very probably a consequence of pulsation.
\end{abstract}

\section{Introduction}

Subdwarf-B $(\mathrm{sdB})$ stars are low-mass $\left(\sim 0.5 M_{\odot}\right)$ core helium burning objects that belong to the Extreme Horizontal Branch (EHB) (Heber 1986). They have a thin and mostly inert hydrogen-rich envelope, and remain hot $(20000 \mathrm{~K} \leqslant$ $\left.T_{\text {eff }} \leqslant 40000 \mathrm{~K}\right)$ and compact $(5 \leqslant \log g \leqslant 6.2)$ throughout their EHB lifetime (Saffer et al. 1994), before evolving towards the white dwarf cooling sequence without experiencing the Asymptotic Giant Branch and Planetary Nebula phases of stellar evolution. Yi et al. (1997) suggest sdB stars could be responsible for the ultraviolet-upturn seen in the energy distributions of elliptical galaxies and spiral galaxy bulges, and therefore understanding their formation may provide an important diagnostic for studying galaxy formation and evolution. Han et al. $(2002,2003)$ use binary population synthesis calculations to demonstrate the formation of sdB stars through several possible channels, but resulting models should still be compared with observation. This could be achieved thanks to the discovery that some subdwarf-B stars undergo non-radial pulsations, which means asteroseismology could be used to study their internal structure. 
There are two main types of pulsating sdB stars: V361 Hya stars are short-period pulsators, and the so-called "Betsy" stars ${ }^{1}$ are long-period pulsators. Kilkenny et al. (1997) serendipitously discovered V361 Hya stars in the Edinburgh-Cape (EC) Blue Object Survey. Typically they undergo short-period $(100-200 \mathrm{~s})$ low-amplitude (a few mmag) multiperiodic pulsations, and they are rather hot and dense $\left(T_{\text {eff }} \sim 34000 \mathrm{~K}, \log g \sim 5.8\right)$. Independently from the observational discovery, pulsating sdB stars were predicted by Charpinet et al. (1996), based on the $\kappa$-mechanism. A local enhancement in the abundance of partially ionised iron in the envelope of sdB stars (due to diffusion in the form of gravitational settling and radiative levitation) causes a bump in the opacity that triggers pulsations. In V361 Hya stars, this results in low-order, low-degree pulsation modes that reach their highest amplitude near the star's surface ( $p$-modes).

Long-period sdB pulsators were discovered by Green et al. (2003). They cluster around $T_{\text {eff }} \sim 25000 \mathrm{~K}, \log g \sim 5.4$, and vary on timescales of about 1 hour, with very low amplitudes, pointing to $g$-mode pulsations. Indeed, Fontaine et al. (2003) demonstrate that the same $\kappa$-mechanism that excites $p$-mode pulsations in V361 Hya stars can also trigger higher degree $g$-mode pulsations in the cooler, less dense "Betsy" stars. Randall et al. (2006a) suggest at least $75 \%$ of cool $\left(T_{\text {eff }}<30000 \mathrm{~K}\right)$ subdwarf-B stars should pulsate with long periods, but so far, only PG $1716+426$ (the class prototype), PG $1627+017$ and PG $1338+481$ have been the subjects of extensive observational efforts, by Reed et al. (2004) and Randall et al. (2006a, 2006b) respectively.

The magnitude of HD 4539 ( $B \sim 10.12$ ) makes it one of the brightest known sdB stars, and it has been extensively studied in the past. For instance, Bascheck et al. (1972) estimate its atmospheric parameters to be $T_{\text {eff }} \sim 25000 \mathrm{~K}$ and $\log g \sim 5.4$, while Saffer et al. (1994) find values of $T_{\text {eff }} \sim 27000 \mathrm{~K}$ and $\log g \sim 5.46$. In any case, this star has surface parameters similar to those of "Betsy" stars. We first observed HD 4539 (2000.0 coordinates: $\left.\alpha=0^{\mathrm{h}} 47^{\mathrm{m}} 29.219^{\mathrm{s}}, \delta=+09^{\circ} 58^{\prime} 55.69^{\prime \prime}\right)$ in August 2005 because inclement weather made it impossible to obtain data on fainter stars. When our spectra exhibited the presence of strong line-profile variations, we decided to perform follow-up observations in subsequent runs to confirm our discovery. In this paper, we therefore report the presence of slow pulsations in HD 4539.

\section{Observations}

Spectroscopic observations were made with the Grating Spectrograph and SITe CCD on the 1.9-m telescope at the South African Astronomical Observatory

\footnotetext{
${ }^{1}$ These are still awaiting official nomenclature, but are informally known as "Betsy" stars, after their discoverer, Dr Elizabeth Green.
} 
(SAAO) in Sutherland, in August 2005, August 2006 and November 2006. Table 1 lists the number of spectra obtained on each night as well as the exposure times.

The typical seeing during our observing runs was rarely better than 1.5", and, accordingly, the typical slit width used was 1.5 " or lower. This setting allowed us to obtain a typical signal-to-noise ratio (SNR) of about 100, without compromising the spectral resolution. Photometry in $\mathrm{B}$ and I was also obtained in 2006 with the UCT CCD and the SAAO CCD on the $0.75-\mathrm{m}$ and 1.0-m Sutherland telescopes respectively. A preliminary analysis shows $\Delta \mathrm{B} \lesssim$ $0.02 \mathrm{mag}$; the details will be reported in a subsequent paper.

Our 198 spectra cover a wavelength range of a little less than $900 \AA$ (from $3950 \AA$ to $4820 \AA$ ) with a resolution of $1 \AA$ and were all obtained alternately with spectra of a $\mathrm{Cu} / \mathrm{Ar}$ arc lamp for wavelength calibration purposes. Reduction was performed using a program specifically written to reduce our data sets automatically. This state-of-the-art code first flat-fields the images before extracting the spectra, then calibrates their wavelength dependence, correcting for the small tilt of the spectra (less than three pixels over a 1798-pixel long chip), and finally subtracts an extensive sky-window, while getting rid of cosmic ray hits and other undesirable effects. The results of our code were compared with reduction performed using IRAF, and the differences were negligible.

In Figure 1, we show a typical spectrum of HD 4539 obtained in August 2005, while Figures 2 and 3 demonstrate the kind of line-profile variations that came to our attention.

Table 1: Observing log for HD 4539.

\begin{tabular}{rrrc}
\hline Date & JD & $\begin{array}{c}\text { Number of } \\
\text { spectra }\end{array}$ & $\begin{array}{c}\text { Integration } \\
\text { time }(\mathrm{s})\end{array}$ \\
\hline 2005 August $23 / 24$ & 2453606 & 1 & 200 \\
$27 / 28$ & 3610 & 16 & 400 \\
$28 / 29$ & 3611 & 11 & 400 \\
$29 / 30$ & 3612 & 28 & 400 \\
2006 August $04 / 05$ & 3952 & 8 & 400 \\
$05 / 06$ & 3953 & 7 & 400 \\
$06 / 07$ & 3954 & 8 & 400 \\
$08 / 09$ & 3956 & 23 & 400 \\
$09 / 10$ & 3957 & 29 & 400 \\
$10 / 11$ & 3958 & 23 & 400 \\
$15 / 16$ & 3963 & 9 & 400 \\
$06 / 07$ & 4046 & 10 & 500 \\
$07 / 08$ & 4047 & 25 & 500 \\
\hline
\end{tabular}




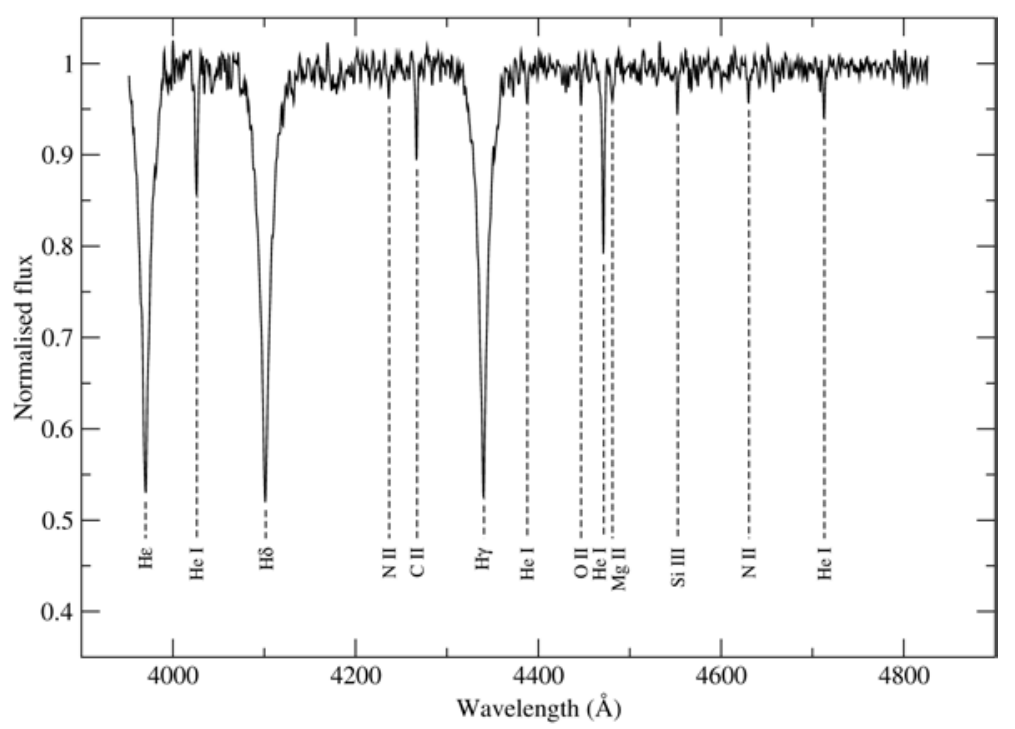

Figure 1: Typical 1.0-Å resolution spectrum of HD 4539, taken at the SAAO 1.9-m telescope in August 2005. Identified lines are labelled.

The fact that the O II and N II lines in the left-hand panels of Figures 2 and 3 exhibit different behaviours (one getting stronger while the other is getting weaker) is similar to the pattern observed by Telting \& Østensen (2004) in the fast pulsator PG $1325+101$, where He II lines are in anti-phase with the other lines. This is an effect of temperature variation due to pulsation, which, in the case of pulsating subdwarf-B stars, cannot be neglected.

Although line-profile variations can be caused by surface spots, this would be very surprising in the case of subdwarf- $B$ stars. Spots can be associated with late-type stars where convection in the atmosphere is important for energy transport, but this is insignificant in sdB stars. It is also likely that abundance spots due to magnetic fields can be ruled out in subdwarf-B stars because they are slow rotators, typically having $v \sin i \leqslant 5 \mathrm{~km} / \mathrm{s}$ (Heber et al. 2000), which corresponds to a rotation period of the order of one day, while periods observed for HD 4539, and variable sdB stars in general, are an order of magnitude smaller and can therefore not arise through a non-uniform abundance distribution in the photosphere consequent upon a magnetic field. On the other hand, Fontaine et al. (2003) demonstrate that non-radial pulsations can be expected in cool subdwarf-B stars like HD 4539 and we investigate this as a possible cause of line-profile variations. 


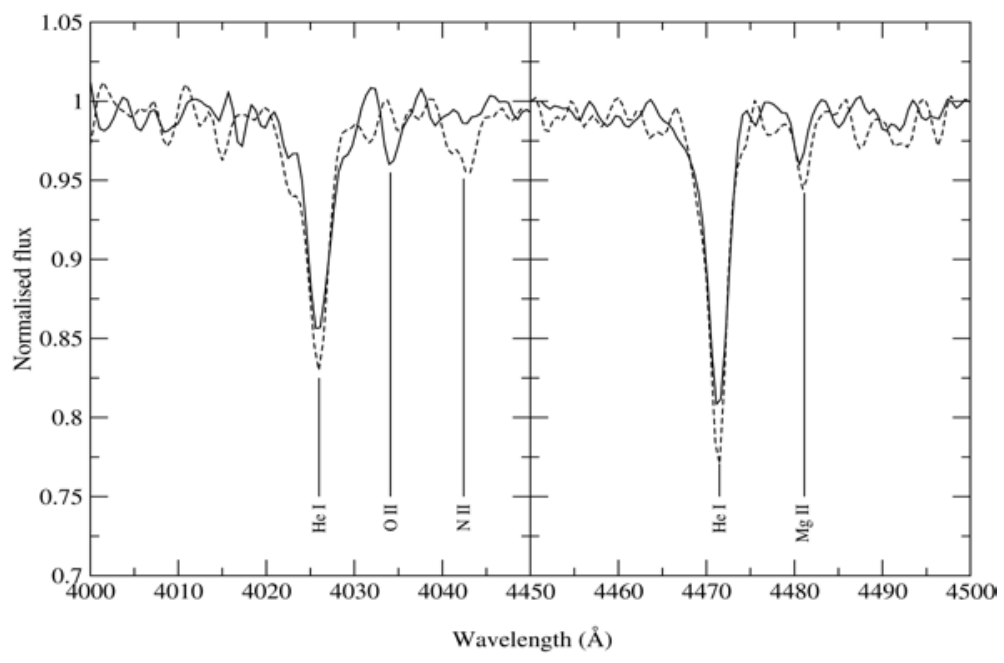

Figure 2: Variations in He I lines of HD 4539 observed on different nights in August 2005. The solid spectrum was obtained on HJD 2453611, and the dashed spectrum on HJD 2453612.

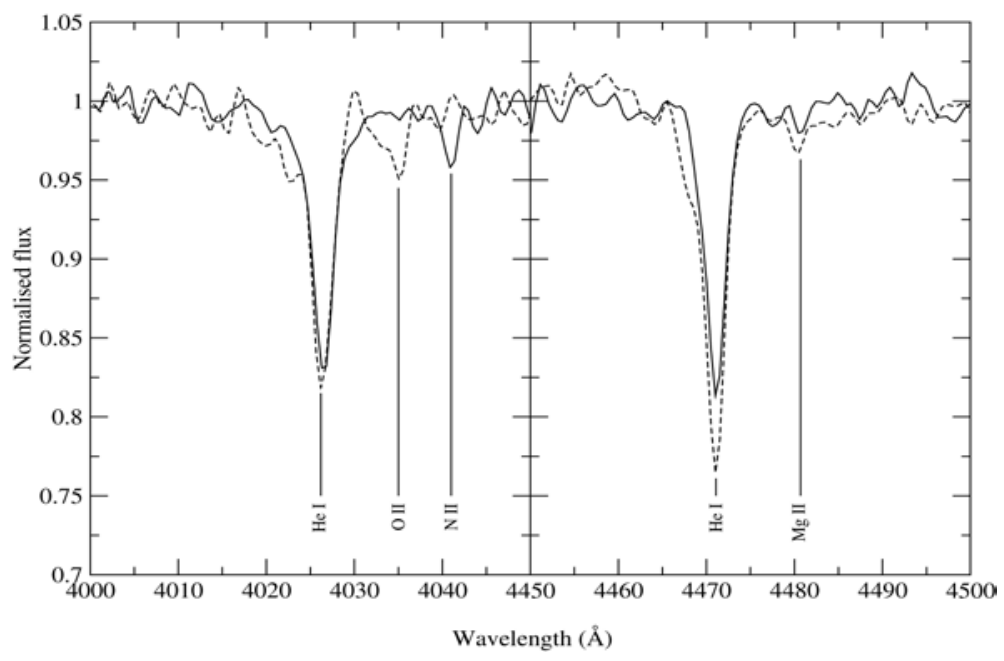

Figure 3: Same as Figure 2, but the solid spectrum was obtained on HJD 2453958, and the dashed spectrum on HJD 2453952. 


\section{Radial velocity analysis}

Radial velocities were derived using VCROSS (Hill 1982), an interactive crosscorrelation program. VCROSS measures radial velocity differences between stars by cross-correlating stellar spectra with one appropriately chosen reference spectrum. Tests on synthetic data by Hill (1982) demonstrate no systematic errors should be expected, and excellent radial velocity determination should be achieved given a proper choice of reference spectrum.

First, we chose our highest signal-to-noise ratio spectrum for each separate night as a reference spectrum, and computed radial velocities for the corresponding nights. We then used the highest SNR spectrum of each run as reference spectrum and obtained radial velocities for each spectrum of the corresponding run. Finally, our best SNR spectrum overall was used to obtain radial velocities for all spectra. This consistency check allowed us to select the spectra with the best determination of radial velocities (about two-thirds of them). After correcting these spectra for the Earth's orbital and diurnal motion, we shifted them to zero radial velocity and added them all together to produce a "super-template" with higher SNR. This template was used to derive new radial velocities for all spectra, and this procedure of shifting the spectra and deriving new radial velocities was to be repeated iteratively until the radial velocities converged (within the fitting errors quoted by VCROSS, that is, less than $0.07 \mathrm{~km} / \mathrm{s}$ ), which, in this particular case, happened after only three iterations. In Figure 4 we plot the radial velocities obtained using the aforementioned method and corrected for the Earth's motion, for each observing night. These were used for the frequency analysis described in the next section.

\section{Frequency analysis}

We performed an extensive frequency analysis of our data set using the software package Period04, described by Lenz \& Breger (2005), and SigSpec, a program providing a rigorous statistical analysis of the significance levels in an amplitude spectrum (Reegen 2007). SigSpec computes a significance spectrum for each detected signal so that, for instance, a significance level of 8 means the considered amplitude would be due to noise in one out of $10^{8}$ cases. It is ideal to deal with strongly aliased data such as ours, when the usual signal-tonoise criteria can become doubtful. Following Reegen's (2007) Equation 31, a signal-to-noise ratio of 4 roughly corresponds to a significance level of 5.4575 , and a signal-to-noise ratio of 3 to a significance of 3.0698 .

When computing the significance spectrum of our data set, we found it be dominated by three low frequency signals (Figure 5, upper panel). This is not 


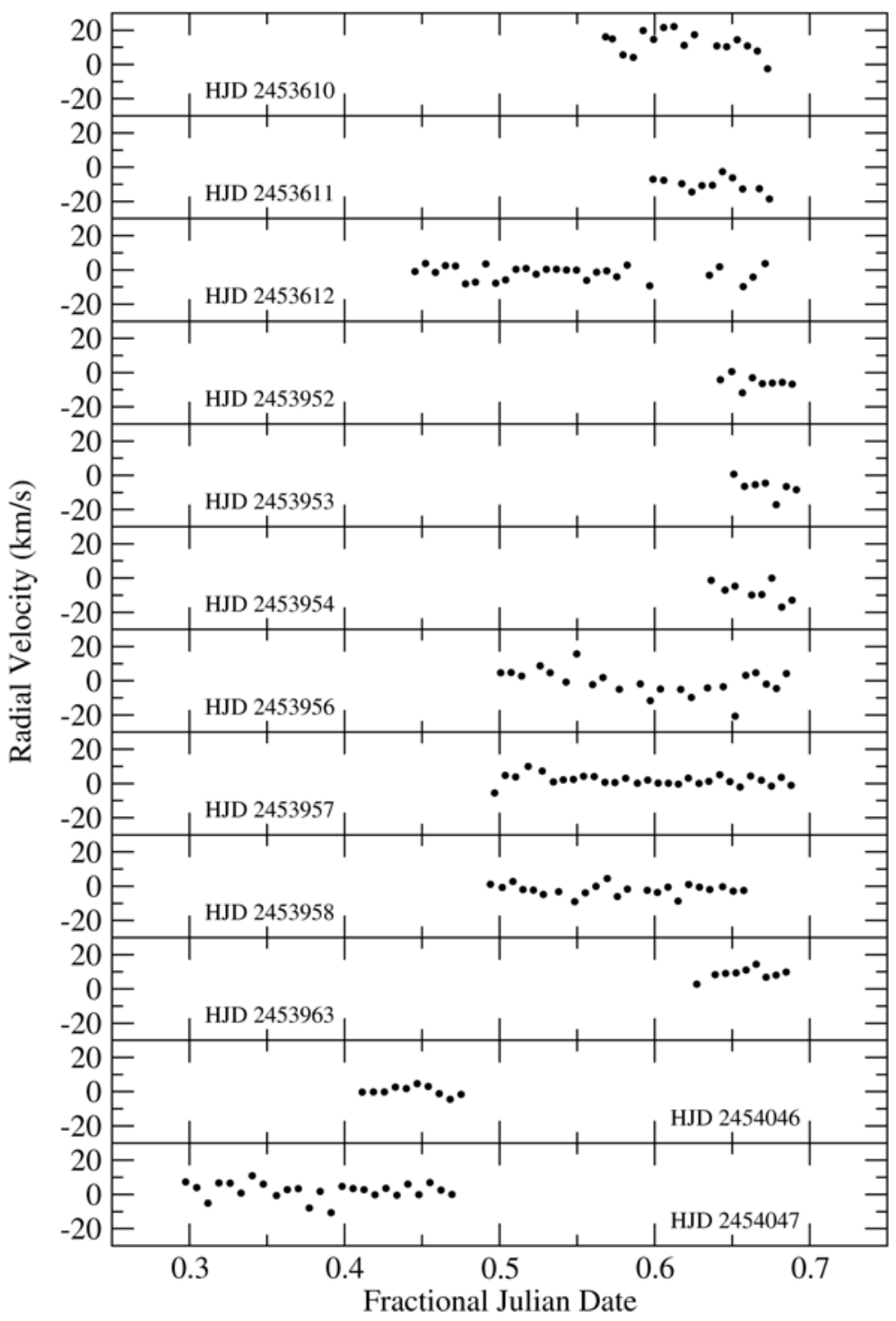

Figure 4: Radial velocities in HD 4539 spectra for each observing night. In particular note the smooth radial velocity variation obtained on HJD 2454046 indicating a typical error of $2 \mathrm{~km} / \mathrm{s}$ 


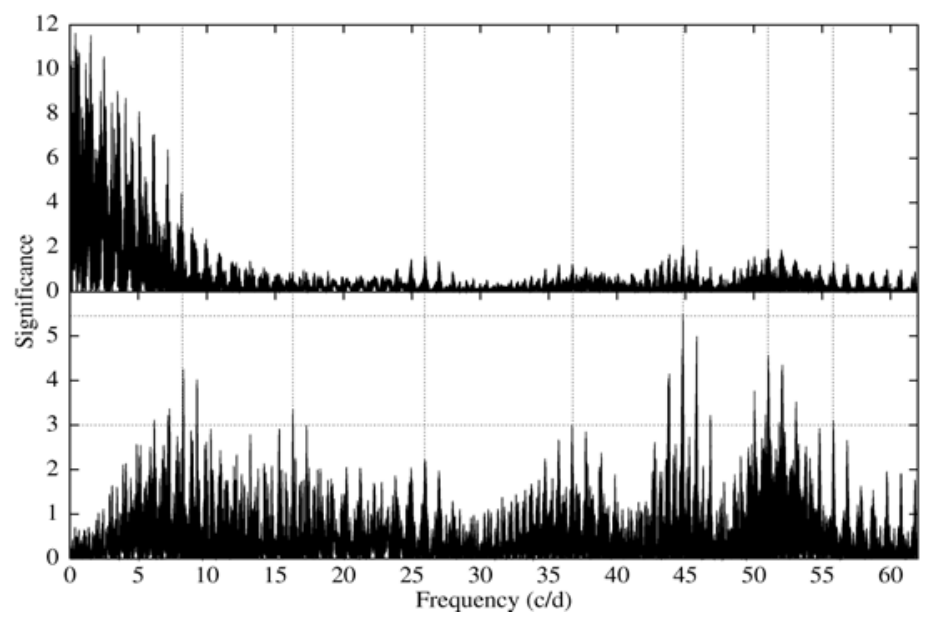

Figure 5: Significance spectra extracted from radial velocity curves of Figure 4, before (top) and after (bottom) removing the low-frequency signals. The horizontal dotted lines correspond to a SNR of 3 and 4 . Vertical dotted lines indicate the frequencies of Table 2 .

unusual in slowly pulsating subdwarf-B stars (see e.g. For et al. 2006, and Kilkenny et al. 2007), and in any case some of these low frequencies can be explained by the sampling of our data. There is however some obvious excess in the significance levels at $\sim 25 \mathrm{c} / \mathrm{d}, \sim 35 \mathrm{c} / \mathrm{d}, \sim 45 \mathrm{c} / \mathrm{d}$ and $\sim 50 \mathrm{c} / \mathrm{d}$.

We therefore decided to analyse our data set after removing the three low frequency signals. After recomputing the significance spectrum, we found one frequency with a significance higher than 5.46, and three others well above the 3.07-significance level, as can be seen in Figure 5 (lower panel) ${ }^{2}$.

The values of these frequencies are summarised in Table 2 after having been optimised in a non-linear least-squares fit, taking into account the influence each frequency can have on the others (Schoenaers \& Cuypers 2004).

In Table 2 we also quote three frequencies with lower significance. Although we cannot confirm whether they're real, and would need additional data to do so, we still consider them as tentative identifications as the significance spectrum exhibits some excess power in these regions.

It is to be noted that we also divided our data set in three parts (one for each run), and analysed them separately. Even though it is obvious we do

\footnotetext{
${ }^{2}$ The significance spectra were computed up to the Nyquist frequency but are only plotted up to $62 \mathrm{c} / \mathrm{d}$ because no power was found to be present at higher frequencies.
} 
Table 2: Frequencies, amplitudes and phases of the variations found in the radial velocity curve of HD 4539. The errors quoted on the frequencies come from a MonteCarlo simulation. The last column gives the significance level of the frequencies.

\begin{tabular}{ccccccc}
\hline & $\begin{array}{c}\text { Frequency } \\
(\mathrm{c} / \mathrm{d})\end{array}$ & \multicolumn{2}{c}{ Period } & Amplitude & Phase & Significance \\
& $44.82( \pm 0.03)$ & 1927 & 0.53 & 2.14 & 0.66 & 5.50 \\
\hline$f_{1}$ & $51.049( \pm 0.001)$ & 1692 & 0.47 & 1.86 & 0.35 & 4.47 \\
$f_{2}$ & $53 r s)$ & $(\mathrm{km} / \mathrm{s})$ & & 4.26 \\
$f_{3}$ & $9.2875( \pm 0.0003)$ & 9310 & 2.59 & 1.71 & 0.05 & 3.23 \\
$f_{4}$ & $16.29( \pm 0.07)$ & 5304 & 1.47 & 1.46 & 0.57 & 2.23 \\
\hline$f_{5}$ & $25.9( \pm 0.1)$ & 3336 & 0.93 & 1.46 & 0.80 & 3.09 \\
$f_{6}$ & $55.807( \pm 0.006)$ & 1548 & 0.43 & 1.40 & 0.48 & 2.93 \\
$f_{7}$ & $37.16( \pm 0.02)$ & 2325 & 0.65 & 1.36 & 0.39 & \\
\hline
\end{tabular}

not have enough data points in the first and third runs to discriminate against some frequencies found in the second run just based on their absence in the first and/or third run, it is nevertheless a worthwhile check to compare the three frequency spectra. Radial velocities obtained in 2006 August gave the same frequencies as the complete dataset, with amplitudes and phases also in agreement within observational error.

\section{Summary}

After three observing runs in 2005 and 2006, we report the discovery of another member of the slowly pulsating subdwarf-B star class. HD 4539 undergoes at least four pulsations with periods ranging from 1692s to 9310s, and amplitudes up to $2.14 \mathrm{~km} / \mathrm{s}$. Being such a bright sdB star, it is an ideal target for an in-depth asteroseismologocal analysis, for example using the method outlined by Schoenaers \& Lynas-Gray (2006). This will be the subject of a subsequent paper.

\section{Acknowledgments.}

The authors wish to thank the SAAO for the generous allocations of observing time, and the University of Oxford, Department of Physics, as well as the United Kingdom Particle Physics and Astronomy Research Council for travel grants. Dr Kilkenny very generously instructed the authors in the use of SAAO facilities and without his help the observations reported here could not have been made. We also wish to thank Dr Reegen for providing us with his code $\mathrm{SigSpec}$, and the anonymous referee for helpful comments. 


\section{References}

Baschek, B., Sargent, W.L.W., \& Searle, L. 1972, ApJ 173, 611

Charpinet, S., Fontaine, G., Brassard, P., \& Dorman, B. 1996, ApJ 471, L103

Fontaine, G., Brassard, P., Charpinet, S., et al. 2003, ApJ 597, 518

For, B.-Q., Green, E. M., O'Donoghue, D., et al. 2006, ApJ 642, 1117-1130

Green, E.M., Fontaine, G., Reed, M.D., et al. 2003, ApJ 583, L31

Han, Z., Podsiadlowski, Ph., Maxted, P.F.L., et al. 2002, MNRAS 336, 449

Han, Z., Podsiadlowski, Ph., Maxted, P.F.L., \& Marsh, T.R. 2003, MNRAS 341, 669

Heber, U. 1986, A\&A 155, 33

Heber, U., Reid, I.N., \& Werner, K. 2000, A\&A 363, 198

Hill, G. 1982, Pub. Dom. Astrophys. Obs. 16, 59

Kilkenny, D., Copley, C., Zietsman, E., \& Worters, H. 2007, MNRAS 375, 1325

Kilkenny, D., Koen, C., O'Donoghue, D., \& Stobie, R.S. 1997, MNRAS 285, 640

Lenz, P., \& Breger, M. 2005, CoAst 146, 53

Randall, S.K., Fontaine, G., Green, E.M., et al. 2006a, ApJ 643, 1198

Randall, S.K., Green, E.M., Fontaine, G., et al. 2006b, ApJ 645, 1464

Reed, M.D., Green, E.M., Callerame, K., et al. 2004, ApJ, 607, 445

Reegen, P. 2007, A\&A 467, 1353

Saffer, R.A., Bergeron, P., Koester, D., \& Liebert, J. 1994, ApJ 432, 351

Schoenaers, C., \& Cuypers, J. 2004, ASPC 310, 283

Schoenaers, C., \& Lynas-Gray, A.E. 2006, BaltA, 15, 219

Telting, J.H., \& Østensen, R. 2004, A\&A 419, 685

Yi S., Demarque, P., Oemler, A.J. 1997, ApJ 486, 201 